\title{
Improvement of renal function by changing the bone-modifying agent from zoledronic acid to denosumab
}

\author{
Mutsushi Yamasaki $^{1} \cdot$ Takeshi Yuasa $^{1} \cdot$ Sho Uehara $^{1} \cdot$ Yasuhisa Fujii $^{1}$ • \\ Shinya Yamamoto ${ }^{1} \cdot$ Hitoshi Masuda $^{1} \cdot$ Iwao Fukui $^{1} \cdot$ Junji Yonese $^{1}$
}

Received: 10 February 2016 / Accepted: 28 June 2016 / Published online: 11 July 2016

(C) Japan Society of Clinical Oncology 2016

\begin{abstract}
Background In order to help in selecting the optimum bone-modifying agent (BMA; zoledronic acid or denosumab), we investigated the impact of the BMA on the renal function of patients with bone metastases.

Materials and methods The present study consisted of 118 patients who were treated with denosumab for bone metastases secondary to prostate cancer, renal cell cancer, and urothelial cancer at our hospital between 2012 and 2015 . The clinical course of the renal function of these patients, treated with zoledronic acid or denosumab, was retrospectively evaluated.

Results Of the 118 patients who were treated with denosumab during the study period, $57(48 \%)$ had previously been administered zoledronic acid and 61 (52\%) had received denosumab as the first-line BMA. The reasons for changing from zoledronic acid to denosumab were increased creatinine serum level (26 patients, $46 \%$ ), patient preference (16 patients, $28 \%$ ), difficulty with venous infusion (10 patients, $17 \%$ ), and other reasons (5 patients, $9 \%$ ). The median level of creatinine clearance in the patients who changed from zoledronic acid to denosumab due to increased serum creatinine level was 59.9 $\mathrm{ml} / \mathrm{min}$ before administration of zoledronic acid, $40.9 \mathrm{ml} /$ min at the beginning of denosumab treatment, $47.5 \mathrm{ml} /$ min at 3 months after administration of denosumab, and $52.0 \mathrm{ml} / \mathrm{min}$ at the last follow-up. There were significant differences.
\end{abstract}

Takeshi Yuasa

takeshi.yuasa@jfcr.or.jp

1 Department of Urology, Cancer Institute Hospital, Japanese Foundation for Cancer Research, Ariake, Tokyo 135-8550, Japan
Conclusions For the first time, we demonstrated that the renal function of some patients, which had deteriorated following zoledronic acid administration, successfully improved after changing to denosumab.

Keywords Zoledronic acid - Denosumab - Renal function · Bone-modifying agents $\cdot$ Bone metastasis

$\begin{array}{ll}\text { Abbreviations } \\ \text { BMA } & \text { Bone-modifying agent } \\ \text { Ccr } & \text { Creatinine clearance ratio } \\ \text { IQR } & \text { Interquartile range } \\ \text { PC } & \text { Prostate cancer } \\ \text { PS } & \text { Performance status } \\ \text { RCC } & \text { Renal cell cancer } \\ \text { UC } & \text { Urothelial cancer }\end{array}$

\section{Introduction}

Bone is a major metastatic site for urologic malignancies as well as many solid tumors. Indeed, prostate cancer (PC) has a very high proclivity for metastasizing to bone, with $\sim 90 \%$ of men with advanced disease having skeletal lesions [1]. In terms of urothelial cancer (UC), bone is also the most common visceral metastatic site from bladder UC, accounting for nearly half of the patients with metastatic UC [2]. In addition, bone is also a common metastatic site in patients with metastatic renal cell cancer (RCC), being second only to the lung, with estimates of frequency ranging from $24-51 \%$ [3]. Bone metastases often create serious clinical problems - they lead to poor performance status due to pathologic fractures, spinal cord compression, and intractable pain, commonly referred to as skeletalrelated events (SREs) [4-9]. Therefore, the diagnosis and 
management of bone metastasis play important roles in the medical treatment of urological malignancies.

In order to prevent SREs associated with bone metastases, the use of bone-modifying agents (BMAs) is currently widespread in urological clinical practice [7-9]. The thirdgeneration bisphosphonate, zoledronic acid $\left(\right.$ Zometa $^{\circledR}$; Novartis Pharma AG, Basel, Switzerland), an inhibitor of bone resorption, is the first authorized BMA, and is one of the standards of care for SREs resulting from metastatic bone lesions in various cancers [4-7]. Denosumab (XGEVA ${ }^{\circledR}$; Amgen, Thousand Oaks, CA, USA) is another BMA that received approval for clinical use in April 2012 in Japan [8-11]. Denosumab is a fully human monoclonal antibody that binds to and neutralizes the receptor activator of nuclear factor-kappa B ligand (RANKL; also called tumor necrosis factor ligand superfamily member 11; TNFSF11), thereby inhibiting osteoclast function and preventing generalized bone resorption and local bone destruction [8-11]. In phase III clinical trials, denosumab was demonstrated to be non-inferior (trending to superior) to zoledronic acid in preventing or delaying first on-study SREs in patients with various malignant tumors, including urological cancers [8-11]. Selecting the optimum BMA, zoledronic acid or denosumab, however, remains unspecified. Renal dysfunction is considered to be one of the main reasons for dose reduction in zoledronic acid therapy [811]. In this study, we report the improvement of renal function by changing the BMA from zoledronic acid to denosumab in patients with urological malignancies.

\section{Materials and methods}

\section{Patients and treatment}

The medical records of patients who were treated with denosumab for bone metastases secondary from prostate cancer (PC), renal cell cancer (RCC), and UC at our institute between 2012 and 2015 were retrospectively reviewed. In order to prevent an effect caused by the deterioration of the primary disease, we included patients treated with denosumab for at least 3 months and excluded patients treated with denosumab for $<3$ months. In all patients, bone metastasis was confirmed by bone scans and computed tomography and/or magnetic resonance imaging. We considered clinical factors, common laboratory blood and serum data, presence or absence of prior zoledronic acid treatment, reason for the change to denosumab, and the clinical course of renal function. The creatinine clearance ratio (Ccr) was calculated according to the method of Cockcroft: $\{[140$ - age (years)] $\times$ weight $(\mathrm{kg})\} /[72 \times$ serum creatinine $(\mathrm{mg} / \mathrm{dl})]$ (multiplied by 0.85 for women and by 1 for men) \}. As for zoledronic acid, dose adjustment as per Ccr is necessary. According to the manufacturer's protocol, $4 \mathrm{mg}$ of zoledronic acid (full dose) can be administered when Ccr is $\geq 60$; however, when $\mathrm{Ccr}$ is $60>\mathrm{Ccr} \geq 50,50>\mathrm{Ccr} \geq 40$, and $40>\mathrm{Ccr} \geq 30$, a reduced dose of $3.5,3.3$, and $3.0 \mathrm{mg}$ of zoledronic acid, respectively, should be administered in clinical practice [12]. This study was carried out in compliance with the Declaration of Helsinki and was approved by the institutional review board at the Cancer Institute Hospital.

\section{Statistical analysis}

The duration of follow-up was calculated from the date of initial administration of BMA to the last administration or the last follow-up. The difference in renal function was compared using Student's $t$ test. Factors associated with changes in renal function were extracted by logistic regression analysis. Statistical analyses were performed using the Statistical Package for Social Sciences, version 17.0 for Windows (SPSS Inc., Chicago, IL, USA). Two-tailed $p<0.05$ was considered significant.

\section{Results}

\section{Characteristics of patients treated with denosumab}

In the medical records of our institute, we found 118 patients who had been treated with denosumab during the study period. The characteristics of these patients are shown in Table 1. The median age of these patients was 71.5 years
Table 1 Characteristics of patients with bone metastasis who were treated with bonemodifying agents

\begin{tabular}{ll}
\hline Variables & \\
\hline Age (years) & $71.5(64.9-76.7)^{\mathrm{a}}$ \\
Male/female (cases) & $101 / 17$ \\
Disease (cases) renal cell cancer/urothelial cancer/prostate cancer & $22 / 27 / 69$ \\
First-line bone-modifying agent (case) & \\
Zoledronic acid & 57 \\
Denosumab & 61 \\
\hline
\end{tabular}

a Numbers represent median (IQR) 
(intraquartile range [IQR] 64.9-76.7 years). The median follow-up period from administration of denosumab to the last follow-up date was 7.6 months (IQR 4.2-13.2 months). Among these patients, $69(58 \%), 27$ (23\%), and $22(19 \%)$ had PC, RCC, and UC, respectively. Of the 118 patients, $101(86 \%)$ were male, and 17 (14\%) were female. Zoledronic acid had previously been administered to 57 patients $(48 \%)$ and $61(52 \%)$ had received denosumab as the firstline BMA. The median number of zoledronic acid administrations was 15 (IQR 8-27 times) and the median dose of the last administration of zoledronic acid was $3.3 \mathrm{mg} / \mathrm{body}$ (IQR 3.0-4.0).

\section{Renal function of patients treated with denosumab as the first-line BMA}

In the 61 patients who received denosumab as the firstline BMA, the median follow-up period from the administration of denosumab to the last follow-up date was 7.7 months (IQR 4.2-13.3 months). Among these patients, $32(52 \%), 11(18 \%)$, and 18 (30\%) had PC, RCC, and $\mathrm{UC}$, respectively. The median level of $\mathrm{Ccr}$ before, at 3 months after administration of denosumab, and at the last follow-up was $74.6 \mathrm{ml} / \mathrm{min}$ (IQR $51.2-93.2 \mathrm{ml} / \mathrm{min}$ ), $78.8 \mathrm{ml} / \mathrm{min}$ (IQR $57.4-94.3 \mathrm{ml} / \mathrm{min}$ ), and $73.3 \mathrm{ml} / \mathrm{min}$ (IQR 59.2-98.8 $\mathrm{ml} / \mathrm{min}$ ), respectively. There was no statistically significant difference among these renal function levels.

\section{Renal function of patients previously treated with zoledronic acid}

Among the 57 patients who received denosumab as the second-line BMA following zoledronic acid, the median follow-up period from administration of zoledronic acid to the last follow-up date was 6.7 months (IQR 3.7-13.3 months). Among these patients, 37 (65\%), 16 (28\%), and 4 (7\%) had PC, RCC, and UC, respectively. The median number of zoledronic acid administrations was 15 (IQR 8-27 times) and the reasons for changing were increased creatinine serum level (26 patients, $46 \%$ ), patient preference (16 patients, $28 \%$ ), difficulty with venous infusion (10 patients, $17 \%$ ), and other reasons (5 patients, $9 \%$ ). We compared the clinical variables between the patients who changed from zoledronic acid to denosumab due to increased serum level $(n=26)$ and the residual patients $(n=31)$ (Table 2). The median Ccr levels at the initial denosumab administration were 75.4 and $40.9 \mathrm{ml} / \mathrm{min}$, respectively, showing a statistical difference. We found that older age and lower $\mathrm{Ccr}$ level were potential risk factors for renal function deterioration during zoledronic acid therapy. In addition, the median level of Ccr before and after administration of zoledronic acid was $68.0 \mathrm{ml} / \mathrm{min}$ (IQR $51.1-91.1 \mathrm{ml} / \mathrm{min}$ ) and $59.5 \mathrm{ml} / \mathrm{min}$ (IQR $39.7-76.4 \mathrm{ml} / \mathrm{min}$ ), respectively (Fig. 1a). The median levels of Ccr before, at 3 months after administration of denosumab, and at the last followup were $59.5 \mathrm{ml} / \mathrm{min}$ (IQR 39.7-76.4 ml/min), 63.3 (IQR $46.3-92.6 \mathrm{ml} / \mathrm{min}$ ), and $64.4 \mathrm{ml} / \mathrm{min}$ (IQR $48.3-90.5 \mathrm{ml} /$ min), respectively (Fig. 1a). Although the Ccr tended to decrease with zoledronic acid treatment and recover with denosumab treatment, there was no statistically significant difference.

\section{Renal function of patients changing from zoledronic acid to denosumab because of renal function deterioration}

Among 26 patients who changed due to renal function deterioration, 14 (54\%), 9 (35\%), and 3 (11\%) had PC, $\mathrm{RCC}$, and UC, respectively. The median number of zoledronic acid treatments was 17.5 (IQR 15-27.5) and the median number of denosumab treatments was 9.5 (IQR 5.25-13.75). The median level of Ccr before and after administration of zoledronic acid was $59.9 \mathrm{ml} / \mathrm{min}$ (IQR $46.9-73.9 \mathrm{ml} / \mathrm{min}$ ) and $40.9 \mathrm{ml} / \mathrm{min}$ (IQR $33.3-55.0 \mathrm{ml} /$ min), respectively (Fig. 1b), showing a statistically significant difference $(p<0.001)$.

Table 2 Comparison of clinical variables between patients who showed deterioration of renal function during zoldronic acid therapy and patients who changed to denosumab due to other causes

\begin{tabular}{llll}
\hline Variables & Deteriorated renal function & Other causes & Probability \\
\hline Creatinine clearance rate at denosumab induction (ml/min) & $40.9(33.3-55.0)$ & $75.4(61.4-103.0)^{\mathrm{a}}$ & $<0.0001$ \\
Age (years) & $74.6(71.4-79.2)^{\mathrm{a}}$ & $68.3(64.1-75.1)^{\mathrm{a}}$ \\
Male/female (cases) & $23 / 3$ & $27 / 4$ & 0.007 \\
Disease (cases) renal cell cancer/urothelial cancer/prostate cancer & $9 / 3 / 14$ & $7 / 1 / 23$ & 0.772 \\
Creatinine clearance rate at zoledonic acid induction (ml/min) & $59.5(45.3-73.5)^{\mathrm{a}}$ & $78.2(58.3-93.3)^{\mathrm{a}}$ & 0.336 \\
Number of zoledronic acid administrations & $17.5(15-27.5)^{\mathrm{a}}$ & $14(6.3-32.3)^{\mathrm{a}}$ & 0.980 \\
\hline
\end{tabular}

${ }^{a}$ Numbers represent median (inter-quartile range) 


\section{A (ml/min)}

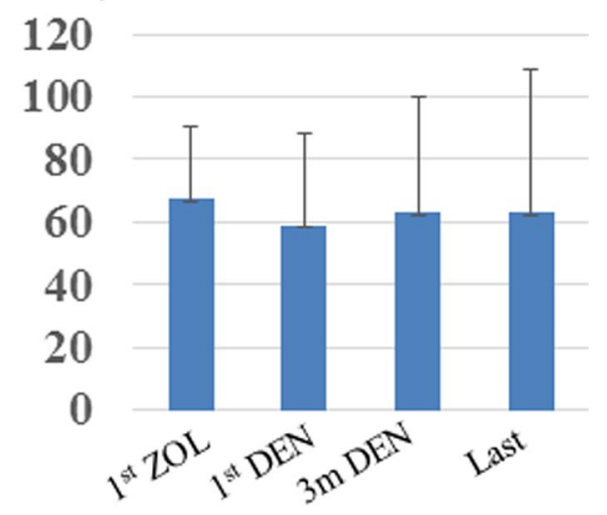

B (ml/min)

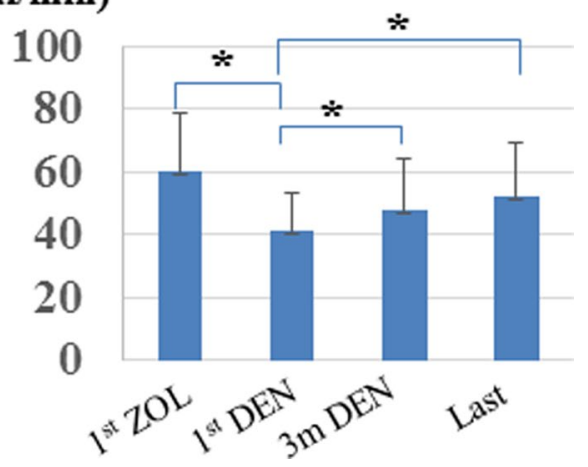

Fig. 1 Changes in the creatinine clearance ratio in patients treated by zoledronic acid followed by denosumab therapy. Renal function of all patients who changed from zoledronic acid to denosumab for any cause (a). Renal function of patients who changed from zoledronic acid to denosumab due to deterioration of renal function (b). $Z O L$ zoledronic acid, $D E N$ denosumab, *statistically significant difference between values $(p<0.001)$

The median levels of $\mathrm{Ccr}$ at the beginning of denosumab treatment, 3 months after the administration of denosumab, and at the last follow-up were $40.9 \mathrm{ml} / \mathrm{min}$ (IQR 33.3-55.0 ml/min), $47.5 \mathrm{ml} / \mathrm{min}$ (IQR 36.5-61.7 ml/ $\mathrm{min}$ ), and $52.0 \mathrm{ml} / \mathrm{min}$ (IQR $41.5-62.8 \mathrm{ml} / \mathrm{min}$ ), respectively (Fig. 1b). The Ccr significantly recovered 3 months after denosumab treatment and at last follow-up $(p<0.001$, $p<0.001$, respectively). In addition, the median increased Ccr during denosumab therapy was $10.0 \mathrm{ml} / \mathrm{min}$ (IQR $3.0-15.8 \mathrm{ml} / \mathrm{min}$ ). When we divided these patients into two groups according to changes in Ccr, the Ccr levels at last follow-up of the patients whose Ccr improved by $>10 \mathrm{ml} /$ min and of the residual patients $(<10 \mathrm{ml} / \mathrm{min})$ were 58.0 (IQR 48.4-74.8 $\mathrm{ml} / \mathrm{min}$ ) and 45.0 (IQR 36.0-50.3 $\mathrm{ml} / \mathrm{min}$ ), respectively, showing a statistical difference $(p=0.012)$. We then compared the clinical variables between these two groups including age, gender, disease, administration times of zoledronic acid, $\mathrm{Ccr}$ at initial zoledronic acid administration, and Ccr at initial denosumab administration; however, there was no statistical difference between the groups.

\section{Discussion}

For patients with bone metastasis, zoledronic acid or denosumab is administered as a BMA to prevent SREs. To date, two classes of pharmaceutic agents, zoledronic acid and denosumab, have received approval by regulatory agencies for use in the treatment of patients with bone metastasis. Denosumab has several beneficial points compared with zoledronic acid, namely, the convenience of subcutaneous administration and the fact that strict renal function monitoring and dose adjustment are not needed. Indeed, during this study period, 57 patients switched from zoledronic acid to denosumab. Increased creatinine serum level (26 patients $46 \%$ ), patient preference (16 patients $28 \%$ ), and difficulty with venous infusion (10 patients $17 \%$ ) were the main reasons for the change.

Renal toxicity caused by zoledronic acid is a well-known adverse event. Post-marketing surveillance has revealed a number of cases of renal failure with zoledronic acid [13, 14]. In 2003, the United States Food and Drug Administration (FDA) reported 72 cases of renal failure associated with zoledronic acid, including 27 patients who required dialysis [13]. Consequently, zoledronic acid requires a dose adjustment for a Ccr of 30-60 ml/min, as described in the 'Materials and methods' section. In this study, due to deteriorated renal function, 26 of 57 (46\%) patients changed from zoledronic acid to denosumab. This renal toxicity leads to reduced relative dose intensity and might reduce the drug's efficacy as a BMA.

Three large international double-blind studies compared denosumab with zoledronic acid for the treatment of bone metastases in patients with castration-resistant prostate cancer $(n=1,904)$, breast cancer $(n=2,046)$, and other advanced cancers or multiple myelomas $(n=1,776)$ [8-10]. The superior results of denosumab in these clinical trials were considered to arise from the difference in relative dose intensity. Among the patients treated with zoledronic acid in these three studies, $502(18 \%)$ had dose adjustments at baseline due to impaired Ccr. In addition, during the study, $277(10 \%)$ patients had doses withheld due to a decrease in Ccr. Moreover, among these 277 patients, 143 (52\%) had PC [14]. In urological malignancies, including RCC and UC of the upper urinary tract, many patients have only one kidney due to prior nephrectomy. As such, urologists must pay careful attention to renal function when treating urological malignancies. In this study, we were unable to compare the efficacy between the zoledronic acid and denosumab. However, we demonstrated that the renal function of some patients treated with zoledronic acid deteriorated during the study period. Older age and lower Ccr might be potential risk factors during zoledronic acid therapy.

In contrast, in terms of administration of denosumab for patients with impaired renal function, the renal function did not have a significant effect on denosumab 
pharmacokinetics or pharmacodynamics [15]. In this study, we discontinued zoledronic acid for renal functionimpaired patients and commenced denosumab therapy immediately. None of the 27 patients who had deteriorated renal function and then started denosumab treatment exhibited a decreased $\mathrm{Ccr}$ during denosumab therapy. Therefore, we can recommend initiation of denosumab treatment and suspension of zoledronic acid when patients exhibit impaired renal function during zoledronic acid treatment. Our data also demonstrated some cases in which renal function was improved by changing from zoledronic acid to denosumab.

The major limitations of our study were the retrospective design and the small size of the study cohort. In addition, we cannot compare the efficacy between zoledronic acid and denosumab in terms of preventing SREs. To date, no report has clearly recommended that changing to denosumab can be a useful choice. We believe that these results reflect the treatment characteristics in patients with bone metastases in current clinical practice in urological malignancies.

In conclusion, we demonstrated for the first time that the renal function of some patients, which had deteriorated following zoledronic acid administration, successfully improved after changing to denosumab. Zoledronic acidcaused renal function deterioration is one of the main reasons for the relative dose intensity reduction as a BMA. We recommend that denosumab be used in patients who experience reduced renal function following zoledronic acid administration.

Acknowledgments The work was partly supported by the Smoking Research Foundation and Grants-in-Aid for Scientific Research from the Ministry of Education, Culture, Sports, Science and Technology, Japan.

\section{Compliance with ethical standards}

Conflict of interest $\mathrm{T}$. Yuasa received remuneration for a lecture from Pfizer Japan (Tokyo, Japan) and Astellas Pharma (Tokyo, Japan). The other authors have declared no conflict of interest.

\section{References}

1. Scher HI, Halabi S, Tannock I, Prostate Cancer Clinical Trials Working Group et al (2008) Design and end points of clinical trials for patients with progressive prostate cancer and castrate levels of testosterone: recommendations of the Prostate Cancer Clinical Trials Working Group. J Clin Oncol 26:1148-1159

2. Shinagare AB, Ramaiya NH, Jagannathan JP et al (2011) Metastatic pattern of bladder cancer: correlation with the characteristics of the primary tumor. Am J Roentgenol 196:117-122

3. Yuasa T, Urakami S, Yamamoto S et al (2011) Treatment outcome and prognostic factors in renal cell cancer patients with bone metastasis. Clin Exp Metastasis 28:405-411

4. Saad F, Gleason DM, Murray R, Zoledronic Acid Prostate Cancer Study Group et al (2002) A randomized, placebo-controlled trial of zoledronic acid in patients with hormone-refractory metastatic prostate carcinoma. J Natl Cancer Inst 94:1458-1468

5. Rosen LS, Gordon D, Tchekmedyian S et al (2003) Zoledronic acid versus placebo in the treatment of skeletal metastases in patients with lung cancer and other solid tumors: a phase III, double-blind, randomized trial-the Zoledronic Acid Lung Cancer and Other Solid Tumors Study Group. J Clin Oncol 21:3150-3157

6. Kohno N, Aogi K, Minami $\mathrm{H}$ et al (2005) Zoledronic acid significantly reduces skeletal complications compared with placebo in Japanese women with bone metastases from breast cancer: a randomized, placebo-controlled trial. J Clin Oncol 23:3314-3321

7. Yuasa T, Kimura S, Ashihara E et al (2007) Zoledronic acid-a multiplicity of anti-cancer action. Curr Med Chem 14:2126-2135

8. Stopeck AT, Lipton A, Body JJ et al (2010) Denosumab compared with zoledronic acid for the treatment of bone metastases in patients with advanced breast cancer: a randomized, doubleblind study. J Clin Oncol 28:5132-5139

9. Fizazi K, Carducci M, Smith M et al (2011) Denosumab versus zoledronic acid for treatment of bone metastases in men with castration-resistant prostate cancer: a randomised, double-blind study. Lancet 377:813-822

10. Henry DH, Costa L, Goldwasser F et al (2011) Randomized, double-blind study of denosumab versus zoledronic acid in the treatment of bone metastases in patients with advanced cancer (excluding breast and prostate cancer) or multiple myeloma. J Clin Oncol 29:1125-1132

11. Yuasa T, Yamamoto S, Urakami S et al (2012) Denosumab: a new option in the treatment of bone metastases from urological cancers. Onco Targets Ther 5:221-229

12. Smith MR, Coleman RE, Klotz L et al (2015) Denosumab for the prevention of skeletal complications in metastatic castrationresistant prostate cancer: comparison of skeletal-related events and symptomatic skeletal events. Ann Oncol 26:368-374

13. Gartrell BA, Coleman RE, Fizazi K et al (2014) Toxicities following treatment with bisphosphonates and receptor times new romanactivator of nuclear factor- $\mathrm{B}$ ligand inhibitors in patients with advanced prostate cancer. Eur Urol 65:278-286

14. Chang JT, Green L, Beitz J (2003) Renal failure with the use of zoledronic acid. N Engl J Med 349:1676-1679

15. Block GA, Bone HG, Fang L et al (2012) A single-dose study of denosumab in patients with various degrees of renal impairment. J Bone Miner Res 27:1471-1479 